\title{
Fractional Derivative based Echo Cancellation for Enhancement of Voice Quality
}

\author{
Abid Khan
}

\begin{abstract}
Echo cancellation and echo suppression are the methods to improve the voice quality. For echo cancellation the right path of echo is necessary. When we transmit a sound signal it is severely effected by echo. In this research paper focus is given to fractional derivative based adaptive strategies for echo cancellation. Because the overall performance of fractional derivative based approach is better than other conventional methods of echo cancellation. Other conventional algorithms for echo cancellation are LMS (least mean square), RLS (recursive least square), and NLMS (normalized least mean square). Where as FNMLS (fractional normalized least mean square) is fractional derivative based method. Therefore, we will exploit this method to improve the performance of echo cancelation algorithm. Various mathematical rule and methods used for fractional derivative based echo cancellation are Taylor series, Grunawld letnikove method, Roy method, Matsuda method, Riemann Liouville formula and L hopital rule. In this research work I will concentrate on fractional derivative based approach for echo cancellation.
\end{abstract}

\section{General Term}

Echo cancellation, Fractional derivative based approach, Interlacing, Radwan procedure

\section{Keywords}

LMS, NLMS, RLS, FNLMS, ERLE, PDES

\section{INTRODUCTION}

Echo severely effect the transmitted signal. The two parameter on which echo depends are amplitude and time delay of reflected waves. In telecommunication echo is a big issue. Echo distort the transmitted signal. Echo cancellation and suppression are method use to improve the voice quality. Echo suppressor for the first time use for satellite communication. Various algorithms use for Echo cancellation are LMS, RLS, NLMS, FXLMS, FLMS, FXRLS, NRLS (Shah S. S., 2014), (Aslam, 2014), (Shah S. S., 2014). During the design of a system we must consider the problem of echo. We must include echo suppressor and echo cancellation circuit in our system.

The introduction of fractional term in fractional order polynomial increase stability. For cancellation of echo we use Kalman filter and other suppression and cancellation circuit (banesty, 2000), (Jones, 1997), (C.Paleologu, 2013). We reduce error to a minimum value. In case of LMS algorithm more number of iteration is required and system is less stable (S.Ciochin, 2013), (Enzner, 2012), (A.Stenger, 1999), (W.Zhu, 2006).

Least Mean Square have a wide range of application in fractional signal processing (F.Kuech, 2005), (D. Manolakis, 2005), (S.Grant, 2008). For better convergence and stability we will choose step size factor very small (S.Haykin, 1996), (Verges, 2010), (Kalman, 2006).For least mean square algorithm the rate of convergence depends on eigen value
(S.Ciochina, 2013), (Gillorie, IEEE), (W.Kellermann, 2008).Echo is the sound of our own voice when we are talking in telephone. The echo is not problematic if its amplitude is small. If echo interval exceed $25 \mathrm{~ms}$ it becomes audible to the speaker. Echo can be extremely disruptive to conversation. It can degrade voice quality (P.Eneroth, 2001), (J.Benesty, 2010), (L.Chu, 2010), (Y. Jung . J. Lee, 2000), (D.Simon, 2000), (al S. e., IEEE).

Echo have some application in Radar and Sonar. When radar transmit a signal after transmission of signal it wait for echo. Acoustic echo produce when a sound signal reflected from a surface and it is hear when sound signal came to end. Echo degraded the quality of sound, to overcome echo we use echo canceller and echo suppressor (H.Michael, November 2003), (N.Sven, 2005).

When two signal coupled together we will use acoustic echo compressor. Acoustic echo compressor technique are used for the removal of noise from signal and improve voice quality (P.Ahgren, 2005), (al S. e., 2006), (L.Fredric, 2007). In public switching telephone network hybrid echo is produce. Hybrid echo is also due to impendence mismatch or where the conversion from one type circuit to another type circuit (S.Gan), (Batalherio P. M., 2008), (Kim S.G, 2008). So to overcome echo we use various mathematical methods for example Couple burger partial differential equation(PDES) using mesh method, Galerkin method, Runwald letnikove method, Saputo, Collocation method and Riesez method (Batalherio P. M., 2004), (Abadi, 2009), (Duttweiler, 2000).

\section{METHODS AND MATERIALS}

Various methods use for Echo cancellation are LMS , NLMS , RLS and ERLS. We splitting actual and desired signal into small signals. The input and desired signal are first filter into sub band using analysis system. Echo cancellation is then performed on each small signal individually. After which the individual residual error of each sub band are reconstructed into a signal residual error signal using the synthesis system.

$>$ Initially model for the cancellation of echo implemented using least mean square algorithm.

$>$ Initially model for the cancellation of echo implemented using NLMS algorithm.

$>$ Proposed FLMS algorithm will be implemented and comparison performed with LMS.

$>$ A fractional NLMS and some variants will be developed and comparison will be performed with NLMS algorithm.

$>$ Frequency domain and sub band algorithm implementation and comparison with proposed algorithms.

Grünwald Letnikove method to reduce the error between exact and approximate solution

The definition of Grünwald letnikove order derivative is 


$$
\begin{aligned}
& { }_{a}^{G} D_{t}^{v} \mathrm{~s}(z)=\lim _{h \rightarrow 0} z_{h}^{v}=\lim _{h \rightarrow 0} h^{-v} \sum_{u=0}^{m}\left[\begin{array}{c}
-v \\
u
\end{array}\right] z(z-u h) \\
& \left\{\left(\begin{array}{c}
u-a \\
m
\end{array}\right) \sum_{u=0}^{m} \begin{array}{l}
\Gamma(u-v) \\
u(u+1)
\end{array}\right\} \\
& \mathrm{n}_{\mathrm{x}}=\left[\begin{array}{c}
\mathrm{x}_{2}-\mathrm{x}_{1} \\
\mathrm{~h}
\end{array}\right] \text { and } \mathrm{n}_{\mathrm{y}}=\left[\begin{array}{c}
\mathrm{y}_{2}-\mathrm{y}_{1} \\
\mathrm{~h}
\end{array}\right] \\
& \mathrm{d}^{\mathrm{v}} \mathrm{s}(\mathrm{g}, \mathrm{h}) \approx \mathrm{s}(\mathrm{g}, \mathrm{h})+(-\mathrm{u}) \mathrm{s}(\mathrm{g}-1, \mathrm{~h})+\frac{(-\mathrm{u})(-\mathrm{u}+1)}{2} \mathrm{~s}(\mathrm{~g}-2, \mathrm{~h})+ \\
& ++\cdots+\frac{\Gamma(-u+1) s(a-n, b)}{n ! \Gamma(-u+n+1)} \\
& \mathrm{d}^{\mathrm{m}}=\mathrm{s}(\mathrm{g}, \mathrm{h})+(-\mathrm{u}) \mathrm{s}(\mathrm{g}, \mathrm{h}-1)+\frac{(-\mathrm{u})(-\mathrm{u}+1)}{2} \mathrm{~s}(\mathrm{~g}, \mathrm{~h}-2)+ \\
& ((-m)(-m+1))(-m+2)+s \frac{g}{n !(-n+1)}++\Gamma(-m+1) \mathrm{s}(g, h-n)
\end{aligned}
$$

The solution of required equation is given below

$$
\left\{\begin{array}{c}
c_{0}=1 \\
c_{1}=-m \\
c_{n}=\frac{\Gamma(-m+1)}{n !(-m+n+1)}
\end{array}\right\}
$$

Where $\Gamma($ ) is equal to (n-1)!

Riemann Liouville equation is an integral function.

Riemann equation is widely used in fractional derivative based adaptive strategies for echo cancellation. In Riemann

$$
D_{x}^{u} \mathrm{~F}(\mathrm{x}) d x=\mathrm{b} I_{x}^{m-u-1} \mathrm{~F}(\tau) \mathrm{d} t .
$$

Riemann equation in summarized form in equation no 5 is given

$D_{x}^{v} \mathrm{f}(\mathrm{x})=\sum_{k=0}^{m-1} \frac{(x-a)^{k-v}}{\Gamma(k-v+1)} f^{(k) a}+\int_{a}^{x} \frac{f^{(n)(t)}}{\left(x-t^{v-n+1}\right)} \mathrm{d} \tau$.

\section{Analysis of fractional order polynomial for stability of} echo cancellation

A fractional order polynomial $\mathrm{P}(\mathrm{s})$ is given. Using different formula and rule we find out the even and odd roots of the given polynomial.

$$
\begin{aligned}
& \mathrm{P}(\mathrm{s})=s^{9.2}+11 s^{8}+52 s^{7.1}+145 s^{6}+266 s^{5}+331 s^{4}+ \\
& 230 s^{3}+155 s^{2}+49 s+6 \\
& {\left[\begin{array}{l}
-.30904118 .1346145331-155 \\
-.30904118 .1346145331-155 \\
-.30904118 .1346145331-155 \\
-.30904118 .1346145331-155 \\
-.30904118 .1346145331-155
\end{array}\right]\left[\begin{array}{l}
\omega_{1} \\
\omega_{2} \\
\omega_{3} \\
\omega_{4} \\
\omega_{5}
\end{array}\right]}
\end{aligned}
$$

equation $\alpha$ is a complex number. The modified form of Riemann equation is given in equation no 4 . The upper and lower limit of Riemann equation are $\mathrm{u}$ and $\mathrm{x}$.

Table (a)

\begin{tabular}{|c|c|c|}
\hline$\omega_{1 e}=.2061$ & $\omega_{6 e}=1.6234$ & $\omega_{1 o}=.4676$ \\
\hline$\omega_{2 e}=.2061$ & $\omega_{7 e}=2.8584$ & $\omega_{2 o}=.4744$ \\
& & $\omega_{3 o}=.8336$ \\
\hline$\omega_{3 e}=.7509$ & $\omega_{8 e}=4.2520$ & $\omega_{4 o}=1.666$ \\
\hline$\omega_{4 e}=7678$ & $\omega_{9 e}=18.0620$ & $\omega_{5 o}=1.971$ \\
\hline
\end{tabular}

For proper echo cancellation the stability of fractional order polynomial is very necessary.

\section{CONCLUSION}

Echo cancellation have many significance for robust transmission of acoustic signal. Acoustic echo cancellation is capable of the eliminating of acoustic echo in difficult conditions such as unbalanced speech levels, close speaker to mic proximity, background noise, indoor/outdoor environment, reflective room surface, double talk and echo path changes. For acoustic echo cancellation we use fractional derivative based adaptive strategies. Echo path detection is also very important for radar and sonar communication. For under water communication echo is also very important. To reduce the error we use various algorithm for example LMS algorithm, RLS algorithm, NLMS algorithm and FNLMS 
algorithms. The overall performance of FNLMS algorithm is better than other conventional methods of echo cancellation.

\section{ACKNOWLEDGEMENT}

The appreciation of this whole research work goes to our kind supervisor Dr. Tariqullah Jan and co supervisor Dr. Syed Muslim Shah.

\section{REFERENCES}

[1] Fractional normalized filter error least mean square algorithm for application in active noise control. Shah, S.M Samar,Raja M.A.Z. 2014, pp. 973-975.

[2] A new adaptive stratgy to improve online secondary path modeling in active noise control system using fractional signal processing approach . Aslam, M.S,Raja ,M.A.Z. 2014, pp. 433-443.

[3] Fractional order constant modulus blind algorithm with application to channel equalization. Shah, S.M, Samar, Naqvi, S.M.R. 2014, pp. 1702-1704.

[4] Acoustic Signal processing for telecommunication. banesty, S.Gay and J. 2000, IEEE.

[5] Nonlinear echo cancellation for hand free speaker phone . Jones, B.S .Nollert D.L. 1997, IEEE.

[6] Study of the optimal simplified Kalman Filter for echo cancellation. C.Paleologu, J banesty S. Cicochin. 2013, IEEE, pp. 580-584.

[7] Study of the optimal simplified filter for echo cancellation. S.Ciochin, C.Paleolug J.Benesty. 2013, IEEE Trans. , pp. 1539-1549.

[8] State space frequency domain adaptive filtering for non linear acoustic echo cancellation . Enzner, S.Malik and G. 2012, IEEE , pp. 2065-2079.

[9] Adaptation of acoustic echo canceller incorporating a memoryless nonlinearity . A.Stenger, W.Kellermann , Rabenstein. 1999.

[10] Compensation of loudspeaker nonlinearity in acoustic echo cancellation using raised cosine type equation . W.Zhu, H.Dai and. 2006, IEEE, pp. 1190-1194.

[11] Nonlinearity acoustic echo cancellation using adaptive orthoganalized power filter . F.Kuech, A.Mitnacht and W.Kellermann. 2005, IEEE, pp. 105-108.

[12] Statistical and adaptive signal processing spectral estimation signal modeling adaptive filtering and array processing. D. Manolakis, V Angel and S.Kogon. 2005, IEEE.

[13] Novel variable step size nlms algorithm for echo cancellation. S.Grant, M. Iqbal and. 2008, IEEE, pp. 241244.

[14] Adaptive filter theory. S.Haykin. 1996, Prentice Hall .

[15] Signal system. Verges, A.V Oppenheim and G.C. 2010.

[16] A new approach to linear filtering and prediction problem . Kalman, R.E. 2006, ASME Journal of basic engineering, pp. 35-45.

[17] Study of the general kalman filter for echo cancellation. S.Ciochina, C. Paleologu J Benesty and. 2013, IEEE, pp. $1539-1549$
[18] Experiement with sub band acoustic echo cancellers for teleconferencing . Gillorie, A. IEEE, 1998, pp. 21412144.

[19] Analysis and design of multi rate system for echo cancellation of acoustic echo. W.Kellermann. 2008, ICASP , pp. 2570-2573.

[20] A real time implementation of a sterphonic acoustic echo canceller . P.Eneroth, S.Gay, T gansler and J. Banesty. 2001, IEEE, pp. 513-523.

[21] Advances in network and acoustic echo cancellation J.Benesty, T.G Ansler , D.R Morgan , M. Sondhi. 2010, Springer.

[22] Weaver SSB sub band Acoustic Echo canceller. L.Chu, Peter. 2010, IEEE.

[23] A new adaptive algorithm for sterophonic acoustic echo canceller. Y. Jung . J. Lee, Y Park, Due-Hee Youn. 2000, IEEE.

[24] Combined acoustic echo and noise reduction using GVSD-based optimal filtering . D.Simon, M.Moonen and C.Erik. 2000, IEEE.

[25] A Psychoacoustic approach to combined acoustic echo cancellation and noise reduction. al, S.Gustafsson et IEEE, IEEE, p. 2002

[26] Acoustic echo cancellation using digital signal processing . H.Michael. November 2003, IEEE.

[27] A blind approach to joint and acoustic echo cancellation N.Sven, Y.Siow and. 2005, IEEE.

[28] Acoustic echo cancellation and double talk detection using estimated loudspeaker impulse response. P.Ahgren. 2005, IEEE

[29] Low complexity adaptive filtering implementation for acoustic echo cancellation. al, S.Christian et. 2006, IEEE.

[30] Efficient multichannel NLMS implementation for acoustic echo cancellation. L.Fredric. 2007, IEEE.

[31] Improving the convergence of the NLMS algorithm using constrained sub band update. S.Gan, Kong A Lee and Woon. IEEE, pp. 736-739.

[32] Non uniform subband adaptive filtering with critical sampling. Batalherio, Petergalia M.R and P. 2008, IEEE, pp. 565-575.

[33] Alias free sub band adaptive filtering with critical sampling . Kim S.G, CD Yoo and T.Q Nguyen. 2008 , IEEE , pp. 1894-1904.

[34] Filter bank design for subband adaptive filtering structure with critical sampling . Batalherio, Petrgalia M.R and PB. 2004, IEEE, pp. 1194-1202.

[35] Proportionate normalized sub band adaptive filter algorithm for sparse System identification. Abadi, Mohammad Shams Esfand. 2009, pp. 1467-1474.

[36] Proportionate normalized adaptation in echo cancellers. Duttweiler, Donald L. 2000, IEEE, pp. 508-518. 